\title{
The Use of CT Head in Diagnosing Subarachnoid Haemorrhage
}

\author{
Bodapati S', Aslam S', Saiepour $A^{2}$ and \\ Thatuskar $\mathbf{A}^{3}$ \\ ${ }^{1} \mathrm{SHO}$, Lincoln County Hospital, UK \\ ${ }^{2} \mathrm{SHO}$, Hull University Teaching Hospital, UK \\ ${ }^{3}$ Consultant, Lincoln County Hospital, UK \\ *Correspondling author: Shruti Bodapati, SHO, \\ Lincoln County Hospital, UK
}

Received: August 31, 2021; Accepted: September 28, 2021; Published: October 05, 2021

\begin{abstract}
Objectives: The aim of our audit was to assess whether a LP is necessary for a diagnosis of SAH if the CT head is negative within six hours.

Methods: A retrospective analysis of 701 CT heads over 15 days was carried out at Lincoln County hospital from $1^{\text {st }}$ to $15^{\text {th }}$ January 2020. Provisional diagnosis of each CT head request was recorded. Radiologists' reports were analyzed and the positive findings were recorded. Patients who had a provisional diagnosis of SAH and had a negative CT head, were further investigated to check if they had a LP and results were recorded. Timing of the CT heads from the onset of symptoms was recorded.
\end{abstract}

Results: Results showed $7 \%$ of CT heads were requested to rule out SAH, $51 \%$ to rule out other intracranial bleeds and the reminder of requests were for other pathologies. Out of the $7 \%$ requests for $\mathrm{SAH}, 2 \%$ were picked up on the CT scans. The remaining $5 \%$ of the patients all had LP. All of the LPs were negative and showed no signs of a SAH. The timing of CT scans showed that only $32 \%$ of patients with provisional diagnosis of SAH had a CT head within 6 hours. $100 \%$ of patients had the CT head done within 12 hours of presentation.

Conclusion: More confidence is needed in using CT heads if done within six hours from symptom onset. Due to high risks associated with LPs, pretest probability should be high before considering LPs.

Keywords: Subarachnoid haemorrhage; Lumbar puncture; Computerizedtomography

\section{Introduction}

Intracranial bleeds are divided into three main sub-types according to the anatomical relation of the bleed to the layers of the meninges. An epidural haemorrhage is bleeding above the dura and below the cranium, a subdural haemorrhage is bleeding below the dura and above the arachnoid layer [1]. A Subarachnoid Haemorrhage (SAH) refers to bleeding into the subarachnoid space, which consists of cerebrospinal fluid, blood vessels, and cisterns. SAH affects about 8 per 100,000 of the population with a gender predilection of 1.6 with women being at a higher risk [2].

$\mathrm{SAH}$ accounts for about $6 \%$ of stroke but it is essential to have a high degree of suspicion in patients presenting with stroke as it is commonly seen in the younger population with the median age of presentation being 50 years.

The most common presenting symptoms of SAH are a sudden severe headache, classically described as a thunderclap headache, meningism (nausea, vomiting, photophobia, neck stiffness, seizures. $10 \%$ of aneurysmal cases cause sudden death [3].

Most patients that are diagnosed with SAH are found to have a spontaneous rupture of cerebral aneurysms (70\%), with arteriovenous malformations being the next most common contributor to SAH [4].

The diagnosis of SAH is especially trying in alert, stable and neurologically intact patients, as missed or delayed diagnosis can be disastrous with high morbidity and mortality. Fast and accurate diagnosis of $\mathrm{SAH}$ is imperative, the appropriate diagnosis and management can reduce the 1-year mortality substantially from $65 \%$ to $18 \%[5,6]$. Studies have suggested that although mortality can be reduced, up to $50 \%$ of surviving patients may have long-term disability [7].

A patient presenting to the $\mathrm{ED}$ with features suggestive of a $\mathrm{SAH}$ will undergo a Computerized-Tomography (CT) scan according to national guidelines this should be performed within the first 6 hours of onset of symptoms [8].

In a CT scan, an acute haemorrhage in the brain can be identified as a high attenuating lesion in the brain. In the case of a SAH this high attenuation will fill the subarachnoid spaces in the brain and often take shape of the sulci. The high attenuation seen is secondary to the haemoglobin content which itself has a high attenuation-coefficient [9]. In the cases where a haemorrhage might have happened several weeks prior to imaging, the scan bleed would not show up as high attenuation.

If the CT scan is negative, the patient will be investigated for other potential causes of their symptoms. 12 hours after symptom onset a Lumbar Puncture (LP) is indicated for the diagnosis or exclusion of SAH [8].

CSF analysis and evidence of xanthochromia clinches the diagnosis of SAH. 
LP come with their own set of risks which include but are not limited to pain at procedure site, bleeding, headache, infection and not to mention a need for hospital admission [10].

If even after a negative LP the suspicion of SAH remains high, the next investigation to obtain would be a CT Angiogram.

Previous research has shown third generation CT scanners to be nearly $100 \%$ sensitive and specific for picking up SAH if done within six hours [11], therefore it is important to address that the timesensitive CT scan is done 6 hours in all units, and coupled with the appreciation of the sensitivity of CT-head results in detected a SAH within the 6-hour window.

\section{Aim}

We aimed to assess whether a Lumbar Puncture (LP) is an absolute necessary tool for a diagnosis of SAH if a non-contrast CT head done within six hours of the initial presentation is negative.

\section{Methodology}

A retrospective analysis was carried out of 701 cranial CT scans over 15 days at Lincoln county hospital from $1^{\text {st }}$ to $15^{\text {th }}$ January 2020.

The provisional diagnosis of each CT head request, as sent in by the clinician who assessed the patient was recorded.

Timing of the scan from the onset of symptoms was noted.

Radiologists' reports were then analyzed and the positive findings were recorded.

We then checked to see if patients who had negative CT head and a provisional diagnosis of $\mathrm{SAH}$ were admitted to the hospital and if they had a LP.

The LP results of these patients were noted.

\section{Results}

Results showed out of the 701 requested studies, 49 (7\%) of the scans were requested to rule out $\mathrm{SAH}, 351(51 \%)$ to rule out other intracranial bleeds and the reminder $301(42 \%)$ of requests were for other pathologies.

Out of the $7 \%$ requests for $\mathrm{SAH}, 2 \%$ were picked up on the CT scans. The remaining $5 \%$ of the patients were admitted and underwent a LP. All of the LPs were negative and showed no signs of a SAH.

The timing of CT scans showed that only $32 \%$ of patients with provisional diagnosis of SAH had a CT head within 6 hours. $100 \%$ of patients had the CT head done within 12 hours of presentation.

\section{Discussion}

Having only $32 \%$ of patients with a provisional diagnosis of SAH received a CT head within 6 hours reduces the sensitivity of picking up all SAH. However, considering the results, there has been no missed SAH on CT scan, deeming all extra LPs performed as an extra investigation, with risks that could have been avoided. A more thorough discussion with patients is also needed to involve them in this discussion making.

A clinically well patient with a CT head negative for SAH should not require admission considering the risks associated with admission and the costs that the hospital incurs for admission. This is with consideration of the risks of discharging a patient who has had a CT head negative for SAH but has not had an LP to investigate for traces of xanthochromia. Confidence in the scans is imperative in making such decisions that could reduce the overall risks a patient is exposed to.

Emerging technologies and methods of imaging may also prove to further increase confidence in interpreting scans.

This study does not cover the discussion of LPs in situations where patients have not undergone a CT head scan within the appropriate 6-hour window from the onset of symptoms. However previous studies have highlighted the difference in sensitivity when exceeding the 6-hour window [12].

A multi-centered or national scale audit is needed to further investigate the accuracy of CT heads when considering a diagnosis of $\mathrm{SAH}$ and formulating guidelines.

\section{Conclusion}

With the development of more sensitive imaging modalities in recent times it has being proven with convincing evidence, that within 6 hours of the index headache, a non-contrast cranial CT is extremely precise, and a negative report for a CT head done after 6 hours considerably reduces the possibility of SAH.

More confidence is needed in using cranial CT scans done within six hours from symptom onset as a tool for final diagnosis and should be enough evidence to safely discharge a patient back home.

Due to high risks associated with LPs, pretest probability and clinical suspicion should be high before considering a patient as a candidate for LPs.

Other causes of headaches should also be ruled out before considering a patient for lumbar puncture.

This study should be followed up with a multi-centered or national scale audit which can investigate the accuracy of today's scanning and reporting methods regarding SAH. This will aid with formulating guidelines in the investigation and management of SAH.

\section{Advances in Knowledge}

With the development of more sensitive imaging modalities in recent times it has being proven with convincing evidence, that within 6 hours of the index headache, a non-contrast cranial CT is extremely precise, and a negative report for a CT head done after 6 hours considerably reduces the possibility of SAH.

\section{References}

1. Tenny S, Thorell W. Intracranial Hemorrhage. 2021.

2. Determining the Sensitivity of Computed Tomography Scanning in Early Detection of Subarachnoid Hemorrhage. Neurosurgery. 2010.

3. Huang J, van Gelder J. The Probability of Sudden Death from Rupture of Intracranial Aneurysms: A Meta-analysis. Neurosurgery. 2002; 51: 11011107.

4. National Study of Subarachnoid Haemorrhage. 2006

5. Korja M, Kivisaari R, Rezai Jahromi B, Lehto H. Natural History of Ruptured but Untreated Intracranial Aneurysms. Stroke. 2017; 48: 1081-1084.

6. Lantigua H, Ortega-Gutierrez S, Schmidt J, Lee K, Badjatia N, Agarwal S, et 
al. Subarachnoid hemorrhage: who dies, and why?. Critical Care. 2015; 19.

7. Caplan's Stroke. 2016.

8. Subarachnoid haemorrhage caused by a ruptured aneurysm: diagnosis and management. 2021.

9. Triulzi F. Cerebral Hemorrhage: CT and MRI. Rivista di Neuroradiologia. 1990; 3: 39-44.

10. Evans R. Complications of Lumbar Puncture. Neurologic Clinics. 1998; 16 : 83-105.
11. Gee C, Dawson M, Bledsoe J, Ledyard H, Phanthavady T, Youngquist S, et al. Sensitivity of Newer-generation Computed Tomography Scanners for Subarachnoid Hemorrhage: A Bayesian Analysis. The Journal of Emergency Medicine. 2012; 43: 13-18.

12. Marcolini E, Hine J. Approach to the Diagnosis and Management of Subarachnoid Hemorrhage. Western Journal of Emergency Medicine. 2019; 20: 203-211. 